\title{
ONE-BIT ADCS IN WIDEBAND MASSIVE MIMO SYSTEMS WITH OFDM TRANSMISSION
}

\author{
Christopher Mollén ${ }^{(1)}$, Junil Choi ${ }^{(2)}$, Erik G. Larsson $^{(1)}$, and Robert W. Heath Jr. ${ }^{(2)}$ \\ (1) Linköping University, Dept. of Electrical Engineering, 58183 Linköping, Sweden \\ (2) University of Texas at Austin, Dept. of Electrical and Computer Engineering, Austin, TX 78712, USA
}

\begin{abstract}
We investigate the performance of wideband massive MIMO base stations that use one-bit ADCs for quantizing the uplink signal. Our main result is to show that the many taps of the frequency-selective channel make linear combiners asymptotically consistent and the quantization noise additive and Gaussian, which simplifies signal processing and enables the straightforward use of OFDM. We also find that single-carrier systems and OFDM systems are affected in the same way by one-bit quantizers in wideband systems because the distribution of the quantization noise becomes the same in both systems as the number of channel taps grows.
\end{abstract}

Index Terms - massive MIMO, OFDM, one-bit ADCs, quantization, wideband.

\section{INTRODUCTION}

A one-bit Analog-to-Digital Converter (ADC) is the simplest device for quantization of an analog signal into a digital. It is the least power consuming quantizer, since the power consumption of ADCs grows exponentially with the number of bits needed to represent all quantization levels [1]. One-bit ADCs would also simplify the analog front end, e.g., automatic gain control would become trivial. Onebit ADCs just output the sign of the input signal, all other information is discarded. The use of such radically coarse quantization has been suggested for use in massive Multiple-Input Multiple-Output (MIMO) base stations, where the large number of radio chains makes high resolution quantization very power consuming [2]. Recent studies have shown that the performance loss due to the coarse quantization of one-bit ADCs becomes less severe as the number of receiving antennas grows; and, in a massive MIMO base station that has hundreds of antennas, the power saving that comes with one-bit ADCs might well outweigh this performance loss [3-5].

Pioneering work on the performance achievable with one-bit ADCs was done in [6-8]. These works and most studies of onebit ADCs since have focused on narrowband systems that have frequency-flat channels. Whether the results also hold for wideband systems, whose channels more realistically are modeled as frequency selective, is not clear from the cited literature.

Here we highlight that, when the number of channel taps is large, quantization noise due to one-bit ADCs is effectively additive and circularly symmetric Gaussian, and affects a system that uses orthogonal frequency division multiplexing (OFDM) the same way it affects a single-carrier system. As a consequence OFDM can easily be implemented in the same way as in the unquantized system. We

The research leading to these results has received funding from the European Union Seventh Framework Programme under grant agreement number ICT-619086 (MAMMOET), the Swedish Research Council (Vetenskapsrådet) and the National Science Foundation under grant number NSFCCF- 1527079 .

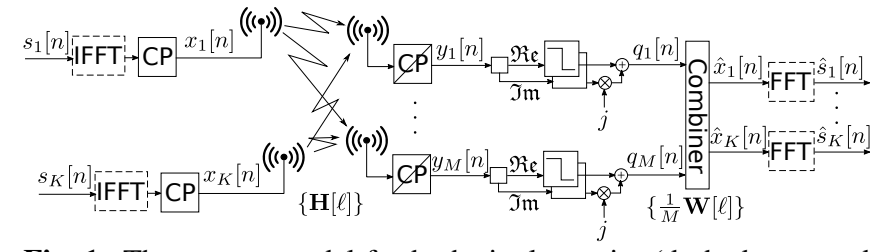

Fig. 1. The system model for both single-carrier (dashed IFFT and FFT are not used) and OFDM transmission (dashed boxes are used).

also show that the averaging effect of having multiple samples from many antennas, which enables the use of one-bit ADCs, is even more effective in a wideband system, where averaging is also done over time due to the frequency selectivity of the channel.

In contrast to the unquantized case, sampling at rates higher than the Nyquist-rate might lead to improved performance in a quantized system [9]. The power consumption of the ADC, however, increases proportionally with the sampling rate [1]. This study is limited to the study of the extreme case, where sampling is done at Nyquist-rate and quantizing is done with one-bit resolution. Related work on loworder ADCs with frequency-selective channels considered nonlinear detection algorithms, either with single-carrier transmission [10] or with OFDM [11]. In contrast, we study a general system that uses simple combiners that are linear in the quantized signals, both for single-carrier and OFDM transmission.

\section{SYSTEM MODEL}

We consider the uplink of the massive MIMO system in Figure 1, where the base station has $M$ antennas and there are $K$ singleantenna users. The system is modeled in complex baseband and the signals are uniformly sampled at the Nyquist-rate with perfect synchronization.

The users transmit the signals $\mathbf{x}[n] \triangleq\left(x_{1}[n], \ldots, x_{K}[n]\right)^{\top}$ at symbol duration $n$ over the frequency-selective channel that is described by the $L$-tap impulse response $\{\mathbf{H}[\ell]\}_{\ell=0}^{L-1}$, where $\mathbf{H}[\ell]$ is an $M \times K$-dimensional matrix. The elements $\left\{h_{m k}[\ell]\right\}_{\ell=0}^{L-1}$ at position $(m, k)$ form the impulse response between user $k$ and base station antenna $m$. To study a wideband scenario, the number $L$ is assumed to be in the order of tens. For example, a system that uses $15 \mathrm{MHz}$ of bandwidth over a channel with $1 \mu$ s of maximum excess delay, which corresponds to a moderately frequency-selective channel, has $L=15$ channel taps, cf. [12], where the "Extended Typical Urban Model" has a maximum excess delay of $5 \mu$ s. The received signal at antenna $m$ at symbol duration $n$ is

$$
y_{m}[n] \triangleq \sum_{k=1}^{K} \sum_{\ell=0}^{L-1} \sqrt{P_{k}} h_{m k}[\ell] x_{k}[n-\ell]+z_{m}[n],
$$

where $z_{m}[n] \sim \mathcal{C N}\left(0, N_{0}\right)$ models the thermal noise of the base station hardware and $P_{k}$ is the transmit power of user $k$.

Copyright 2016 IEEE. Published in the IEEE 2016 International Conference on Acoustics, Speech, and Signal Processing (ICASSP 2016), scheduled for 20-15 March 2016 in Shanghai, China. Personal use of this material is permitted. However, permission to reprint/republish this material for advertising or promotional purposes or for creating new collective works for resale or redistribution to servers or lists, or to reuse any copyrighted component of this work in other works, must be obtained from the IEEE. Contact: Manager, Copyrights and Permissions / IEEE Service Center / 445 Hoes Lane / P.O. Box 1331 / Piscataway, NJ 08855-1331, USA. Telephone: + Intl. 908-562-3966. 
Upon reception, the in-phase and quadrature signals are separately quantized, each by a one-bit ADC:

$$
q\left(y_{m}[n]\right) \triangleq \frac{1}{\sqrt{2}} \operatorname{sign}\left(\mathfrak{R e} y_{m}[n]\right)+j \frac{1}{\sqrt{2}} \operatorname{sign}\left(\mathfrak{I m} y_{m}[n]\right) .
$$

Here the threshold of the one-bit ADC is assumed to be zero. Other thresholds are also possible [13]. The arbitrary scaling of the quantized signal is chosen such that (2) has unit power. For convenience, we denote $q_{m}[n] \triangleq q\left(y_{m}[n]\right)$ and $\mathbf{q}[n] \triangleq\left(q_{1}[n], \ldots, q_{M}[n]\right)^{\top}$.

Both single-carrier and OFDM transmission are studied. The transmission is observed for a block of $N$ symbols. During symbol periods $n=0, \ldots, N-1$, we assume that the users transmit

$$
\mathbf{x}[n]=\left\{\begin{array}{ll}
\mathbf{s}[n], & \text { if single-carrier } \\
\frac{1}{\sqrt{N}} \sum_{\nu=0}^{N-1} \mathbf{s}[\nu] e^{j 2 \pi n \nu / N}, & \text { if OFDM }
\end{array},\right.
$$

where $\mathbf{s}[n]=\left(s_{1}[n], \ldots, s_{K}[n]\right)^{\top}$ is the vector of data symbols that are concurrently transmitted during symbol duration $n$ by the $K$ users. We assume that $\mathrm{E}\left[s_{k}[n]\right]=0$ and $\mathrm{E}\left[\left|s_{k}[n]\right|^{2}\right]=1$ for all $k, n$. The users also transmit an $L-1$-symbol long cyclic prefix:

$$
\mathbf{x}[n]=\mathbf{x}[N+n], \quad-L<n<0 .
$$

The signal power is normalized such that $\mathrm{E}\left[\left|x_{k}[n]\right|^{2}\right]=1, \forall n, k$.

\section{RECEIVER COMBINING}

The base station combines the received signals in a FIR filter to estimate the transmitted signals, just as it would have if the quantization were perfect. The resulting estimate is

$$
\hat{\mathbf{x}}[n] \triangleq\left(\hat{x}_{1}[n], \ldots, \hat{x}_{K}[n]\right)^{\top}=\frac{1}{M} \sum_{\ell=\ell_{\min }}^{\ell_{\max }} \mathbf{W}[\ell] \mathbf{q}[n-\ell],
$$

where $\ell_{\min }$ and $\ell_{\max }$ are the smallest and largest indices of the nonzero taps of the impulse response of the combiner $\left\{\mathbf{W}[\ell] \in \mathbb{C}^{K \times M}\right\}$ :

$$
\mathbf{W}[\ell] \triangleq \frac{1}{N} \sum_{n=0}^{N-1} \tilde{\mathbf{W}}[n] e^{j 2 \pi n \ell / N}, \quad \ell=\ell_{\min }, \ldots, \ell_{\max },
$$

where the frequency domain combining matrices $\tilde{\mathbf{W}}[n]$ can be defined in a number of different ways. Three common linear combiners are the Maximum-Ratio, Zero-Forcing, and Minimum MeanSquare-Error Combiners (MRC, ZFC, MMSEC):

$$
\tilde{\mathbf{W}}[n]=\left\{\begin{array}{ll}
\tilde{\mathbf{H}}^{\mathrm{H}}[n], & \text { if MRC } \\
\left(\tilde{\mathbf{H}}^{\mathrm{H}}[n] \tilde{\mathbf{H}}[n]\right)^{-1} \tilde{\mathbf{H}}^{\mathrm{H}}[n], & \text { if ZFC } \\
\left(\tilde{\mathbf{H}}^{\mathrm{H}}[n] \tilde{\mathbf{H}}[n]+\rho \mathbf{I}_{K}\right)^{-1} \tilde{\mathbf{H}}^{\mathrm{H}}[n], & \text { if MMSEC }
\end{array},\right.
$$

where $\rho$ in the definition of MMSEC is a system parameter that can be used to make the combiner more like MRC (large $\rho$ ) or more like ZFC (small $\rho$ ). The definitions are in terms of the channel spectrum:

$$
\tilde{\mathbf{H}}[n] \triangleq \sum_{\ell=0}^{L-1} \mathbf{H}[\ell] e^{-j 2 \pi n \ell / N} .
$$

The element at position $(k, m)$ of $\mathbf{W}[\ell]$ is denoted by $w_{k m}[\ell]$. The symbol estimates are obtained directly as

$$
\hat{\mathbf{s}}[n]=\left\{\begin{array}{ll}
\hat{\mathbf{x}}[n], & \text { if single-carrier } \\
\frac{1}{\sqrt{N}} \sum_{\nu=0}^{N-1} \hat{\mathbf{x}}[\nu] e^{-j 2 \pi \nu n / N}, & \text { if OFDM }
\end{array} .\right.
$$

In the comparison of single-carrier and OFDM transmission, we assume that the channel is perfectly known to the base station. In reality, perfect channel state knowledge is not realistic in massive MIMO, because of the huge dimension of the channel, and more so when one-bit ADCs are used, due to the coarse quantization of the received signals. We reason that any estimation of the channel will affect a single-carrier system the same way it will affect an OFDM system. Therefore, if the performance of the two systems is the same with perfect channel state information, it should also be the same with imperfect channel state information. Previous work [14,15] has shown that channel state information can be acquired in a massive MIMO system even with one-bit ADCs.

\section{QUANTIZATION NOISE}

By showing that the estimates of the linear combiners in (7) are consistent also with one-bit ADCs, we will show that the orthogonality of the transmit symbols are preserved and that OFDM easily can be implemented in massive MIMO when the number of taps is large.

\subsection{Consistency of Linear Combiners}

The following theorem states that, in the limit of infinite number of antennas, the estimates of linear combiners converge to a value from which the transmit signal can be recovered, i.e., that linear combiners are consistent.

Theorem 1. In a Rayleigh fading channel with uniform delay profile, $h_{m k}[\ell] \sim \mathcal{C N}\left(0, \frac{1}{L}\right)$ IID for all $m, k$ and $\ell$, the linear combiners in (7) are consistent, i.e., given the transmit signals $\left\{x_{k}[n]\right\}$, there exists a deterministic invertible function $g: \mathbb{C} \rightarrow \mathbb{C}$ such that

$$
\hat{x}_{k}[n] \stackrel{\text { a.s. }}{\longrightarrow} g\left(x_{k}[n]\right), \quad M \rightarrow \infty .
$$

Proof. We note that all combiners in (7) converge to the MRC as the number of antennas tends to infinity for an IID Rayleigh fading channel because the propagation is favorable [16]. We therefore consider the MRC estimate of the $k$-th user:

$$
\hat{x}_{k}[n]=\frac{1}{M} \sum_{m=1}^{M} \sum_{\ell=0}^{L-1} h_{m k}^{*}[\ell] q_{m}[n+\ell] .
$$

Since the terms in the sum are IID, by the law of large numbers,

$$
\begin{aligned}
& \lim _{M \rightarrow \infty} \hat{x}_{k}[n]=\sum_{\ell=0}^{L-1} \mathrm{E}\left[h_{m k}^{*}[\ell] q_{m}[n+\ell]\right] \\
& =\sum_{\ell=0}^{L-1} \mathrm{E}\left[h_{m k}^{*}[\ell] \mathrm{E}\left[q\left(\sqrt{P_{k}} h_{m k}[\ell] x_{k}[n]+u_{k}[n, \ell]\right) \mid h_{m k}[\ell]\right]\right],
\end{aligned}
$$

where

$$
u_{k}[n, \ell] \triangleq \sum_{\substack{k^{\prime}=1 \\\left(k^{\prime}, \ell^{\prime}\right) \neq(k, \ell)}}^{K} \sum_{\ell^{\prime}=0}^{L-1} \sqrt{P_{k^{\prime}}} h_{m k^{\prime}}\left[\ell^{\prime}\right] x_{k^{\prime}}\left[n+\ell-\ell^{\prime}\right]+z_{m}\left[n+\ell^{\prime}\right]
$$

We note that $u_{k}[n, \ell] \sim \mathcal{C N}\left(0, I_{k}[n, \ell]\right)$, where

$$
I_{k}[n, \ell] \triangleq \frac{1}{L} \sum_{\substack{k^{\prime}=1 \\\left(k^{\prime}, \ell^{\prime}\right) \neq(k, \ell)}}^{K} \sum_{\ell^{\prime}=0}^{L-1} P_{k^{\prime}}\left|x_{k^{\prime}}\left[n+\ell-\ell^{\prime}\right]\right|^{2}+N_{0} .
$$

It is noted that, for any given complex numbers $h$ and $x$ and stochastic variable $u \sim \mathcal{C N}\left(0, \sigma^{2}\right)$, it is true that

$$
\begin{aligned}
& \sqrt{2} \mathfrak{R e}(\mathrm{E}[q(h x+u) \mid h]) \\
& =\operatorname{Pr}(\mathfrak{R e} h x>-\mathfrak{R e} u \mid h)-\operatorname{Pr}(\mathfrak{R e} h x<-\mathfrak{R e} u \mid h) \\
& =1-2 \operatorname{Pr}(\mathfrak{R e} h x<-\mathfrak{R e} u \mid h) \\
& =1-2 \mathrm{Q}\left(\frac{\sqrt{2} \mathfrak{R e} h x}{\sigma}\right) .
\end{aligned}
$$


The imaginary part can be rewritten in the same way.

We now let $h_{\mathfrak{R e}} \triangleq \mathfrak{R e} h_{m k}[n]$ and $h_{\mathfrak{I m}} \triangleq \mathfrak{I m} h_{m k}[n]$ be the IID real and imaginary parts of the channel coefficient, $h_{\mathfrak{R e}} \sim h_{\mathfrak{I m}_{\mathfrak{m}}} \sim$ $\mathcal{N}\left(0, \frac{1}{2 L}\right)$. To simplify the mathematical notation, we initially assume that $x_{k}[n]$ is real in the following steps-the complex interfering transmit signals $x_{k^{\prime}}\left[n^{\prime}\right],\left(k^{\prime}, n^{\prime}\right) \neq(k, n)$ are still arbitrary. Then the variable $a \triangleq x_{k}[n] \sqrt{\frac{2 P_{k}}{I_{k}[n, \ell]}}$ is real and the expected value in (13) is given by

$$
\begin{aligned}
& \frac{1}{\sqrt{2}} \mathrm{E}\left[\left(h_{\Re \mathfrak{e}}-j h_{\mathfrak{I m}}\right)\left(1-2 \mathrm{Q}\left(a h_{\mathfrak{R e}}\right)+j-j 2 \mathrm{Q}\left(a h_{\mathfrak{I m}}\right)\right)\right] \\
& =-\sqrt{2} \mathrm{E}\left[h_{\mathfrak{R} \mathfrak{e}} \mathrm{Q}\left(a h_{\mathfrak{R} \mathfrak{e}}\right)+h_{\mathfrak{I} \mathfrak{m}} \mathrm{Q}\left(a h_{\mathfrak{I} \mathfrak{m}}\right)\right. \\
& \left.+j\left(h_{\mathfrak{R} \mathfrak{e}} \mathrm{Q}\left(a h_{\mathfrak{I m}}\right)-h_{\mathfrak{I m}} \mathrm{Q}\left(a h_{\mathfrak{R e}}\right)\right)\right] \\
& =-2 \sqrt{2} \mathrm{E}\left[h_{\mathfrak{R} \mathfrak{e}} \mathrm{Q}\left(a h_{\mathfrak{R} \mathfrak{e}}\right)\right] \text {. }
\end{aligned}
$$

In the first step, we used (18) and its imaginary counterpart. In the last step, the two terms of the imaginary part of the expectation are zero, because the real and imaginary parts $h_{\mathfrak{R e}}$ and $h_{\mathfrak{I m}}$ are independent and zero mean, and the two terms of the real part are the same, because they are identically distributed.

If we let $f_{h}(h)$ be the probability density function of a $\mathcal{N}\left(0, \frac{1}{2 L}\right)$ distributed random variable, then (13) becomes

$$
\begin{aligned}
\lim _{M \rightarrow \infty} \hat{x}_{k}[n] & =-2 \sqrt{2} \sum_{\ell=0}^{L-1} \int_{-\infty}^{\infty} f_{h}(h) h \mathrm{Q}\left(x_{k}[n] \sqrt{\frac{2 P_{k}}{I_{k}[n, \ell]}} h\right) \mathrm{d} h \\
& \triangleq g^{\prime}\left(x_{k}[n]\right) .
\end{aligned}
$$

The steps in (19)-(21) can be repeated for $x_{k}[n]$ with arbitrary modulus. It can then be seen that, for a general transmit signal $x_{k}[n]$,

$$
\lim _{M \rightarrow \infty} \hat{x}_{k}[n]=\frac{x_{k}[n]}{\left|x_{k}[n]\right|} g^{\prime}\left(\left|x_{k}[n]\right|\right) \triangleq g\left(x_{k}[n]\right) .
$$

Because of this relation between the functions $g$ and $g^{\prime}$, it is enough to prove that $g^{\prime}: \mathbb{R} \rightarrow \mathbb{R}$ is monotonic to prove that $g$ also is invertible. We consider the derivative of $g^{\prime}$ :

$$
\begin{aligned}
\frac{\mathrm{d}}{\mathrm{d} x} g^{\prime}(x) & =\sum_{\ell=0}^{L-1} \frac{2 \sqrt{2 P_{k}}}{\sqrt{\pi I_{k}[n, \ell]}} \int_{-\infty}^{\infty} f_{h}(h) h^{2} e^{-\frac{P_{k} x^{2} h^{2}}{I_{k}[n, \ell]}} \mathrm{d} h \\
& >0, \quad \forall x .
\end{aligned}
$$

Here we used the fact that $\frac{\mathrm{d}}{\mathrm{d} x} \mathrm{Q}(x)=-\frac{1}{\sqrt{2 \pi}} e^{-\frac{x^{2}}{2}}$. Since $g^{\prime}$ is monotonically increasing, it is invertible. Because of (24), $g$ is invertible too.

Theorem 1 tells us that if $I_{k}[n, \ell]$ is known, then $x_{k}[n]$ can be detected error-free as $M \rightarrow \infty$. Since knowing $I_{k}[n, \ell]$ requires some knowledge of the interfering symbols $\left\{x_{k^{\prime}}\left[n^{\prime}\right]\right\},\left(k^{\prime}, n^{\prime}\right) \neq(k, n)$, determining it perfectly is only possible in a single-user frequencyflat channel, where $I_{k}[n, \ell]=N_{0}$. In a wideband system, however, the function $g$ does not depend on $I_{k}[n, \ell]$, only its mean, and good detection can be achieved without knowledge of the interfering symbols. This is formalized in the following theorem.

Theorem 2. In a wideband system, the function $g$ approaches the deterministic linear function

$$
g\left(x_{k}[n]\right) \stackrel{\text { a.s. }}{\longrightarrow} \sqrt{\frac{2}{\pi}} x_{k}[n] \sqrt{\frac{P_{k}}{N_{0}+\sum_{k^{\prime}=1}^{K} P_{k^{\prime}}}}, \quad L \rightarrow \infty .
$$
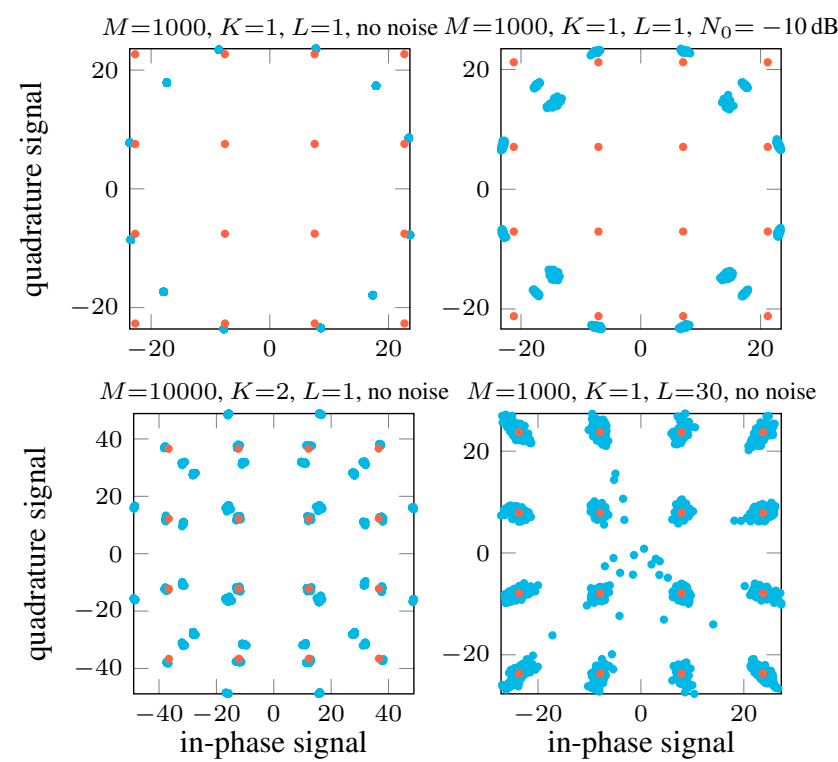

Fig. 2. The MRC estimates (blue) and the 16-QAM transmit signals (red) with $M$ antennas, $K$ users, $L$ channel taps. The power $P_{k}=1$, $\forall k$, and the channel is IID Rayleigh fading $h_{m k}[\ell] \sim \mathcal{C N}\left(0, \frac{1}{L}\right)$. The channel is known at the base station.

Proof. First, we note that the interference becomes deterministic:

$$
I_{k}[n, \ell] \stackrel{\text { a.s. }}{\longrightarrow} N_{0}+\sum_{k^{\prime}=1}^{K} P_{k^{\prime}}, \quad L \rightarrow \infty .
$$

By evaluating the integral in (25), it is seen that the derivative converges pointwise:

$$
\begin{aligned}
& \frac{\mathrm{d}}{\mathrm{d} x} g^{\prime}(x)=\sqrt{\frac{2}{\pi}} \sum_{\ell=0}^{L-1} \sqrt{\frac{P_{k}}{I_{k}[n, \ell]}} \frac{\sqrt{L}}{\left(L+\frac{P_{k} x^{2}}{I_{k}[n, \ell]}\right)^{3 / 2}} \\
& \stackrel{\text { a.s. }}{\longrightarrow} \sqrt{\frac{2 P_{k}}{\pi\left(N_{0}+\sum_{k^{\prime}=1}^{K} P_{k^{\prime}}\right)}}, \quad L \rightarrow \infty .
\end{aligned}
$$

The sum in (29) is dominated by the constant function $\sqrt{\frac{P_{k}}{I_{\min }}}$, where $I_{\min } \triangleq \min \left\{I_{k}[n, \ell], L=1, \ldots\right\}$, for any realization $\left\{x_{k}[n]\right\}$, i.e.,

$$
\sum_{\ell=0}^{L-1} \sqrt{\frac{P_{k}}{I_{k}[n, \ell]}} \frac{\sqrt{L}}{\left(L+\frac{P_{k} x^{2}}{I_{k}[n, \ell]}\right)^{3 / 2}} \leq \sqrt{\frac{P_{k}}{I_{\min }}}, \quad \forall L .
$$

Because the dominating function is integrable over any finite interval $[0, x]$, the limit of $g^{\prime}(x)$ can be obtained by integration of the limit of its derivative (30) according to the theorem of dominated convergence. Because $g^{\prime}(0)=0$, this completes the proof.

The consistency and the behavior of the function $g$ can be seen in the examples given in Figure 2. In the upper left system, no amplitude information can be recovered, even if the number of antennas is large, because the variance $I_{k}[n, \ell]=0$. In this case, dithering the received signal prior to quantization helps in recovering the amplitude, see the upper right system, where noise has been added. The function $g$ is nonlinear but amplitude information is still recoverable with enough antennas at the base station. In the lower left system, the variance $I_{k}[n, \ell]$ assumes three distinct values depending on the power of the transmit signal of the second user, which results in three possible points for each symbol estimate. In the lower right wideband system however, the function $g$ is deterministic and linear. 

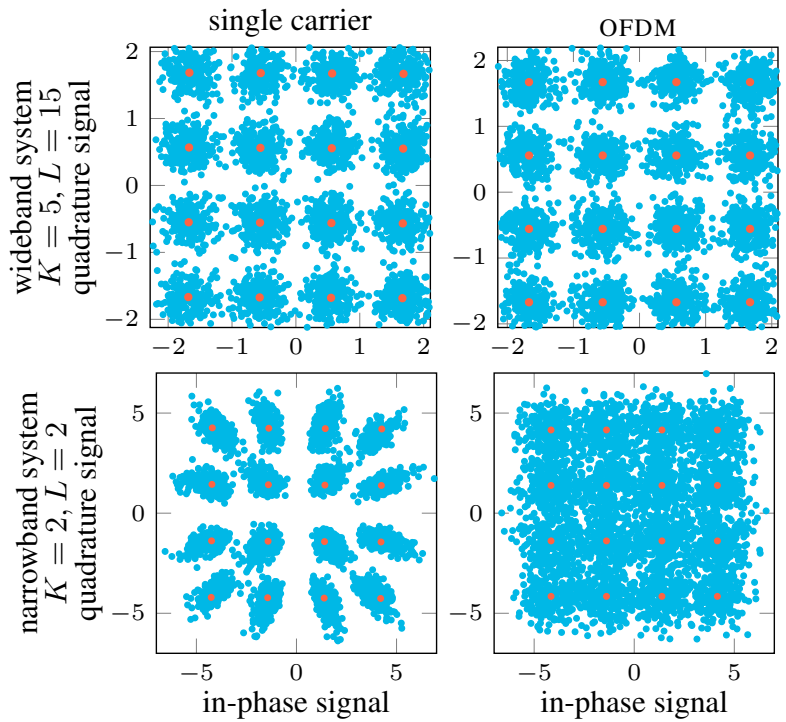

Fig. 3. Symbol estimates $\hat{s}_{k}[n]$ after one-bit quantization and ZFC in a massive MIMO base station with 128 antennas that serves $K$ users over an $L$-tap Rayleigh fading channel, $P_{k}=1, \forall k$, and $N_{0}=0$.

\subsection{Distribution of Error Due to Quantization}

The error after quantization is given by:

$$
e_{k}[n] \triangleq \hat{x}_{k}[n]-\mathrm{E}\left[g\left(x_{k}[n]\right) \mid x_{k}[n]\right]=d_{k}[n]+r_{k}[n],
$$

where

$$
\begin{aligned}
& d_{k}[n] \triangleq \frac{1}{M} \sum_{m=1}^{M} \sum_{\ell=0}^{L^{\prime}-1} w_{k m}[\ell] q_{k}[n-\ell]-g\left(x_{k}[n]\right) \\
& r_{k}[n] \triangleq g\left(x_{k}[n]\right)-\mathrm{E}\left[g\left(x_{k}[n]\right) \mid x_{k}[n]\right] .
\end{aligned}
$$

The first term becomes circularly symmetric Gaussian when the number of antennas grows large according to the central limit theorem. The second term is a term that is proportional to the transmit signal $x_{k}[n]$. The error, thus, consists of two parts: one circularly symmetric Gaussian $d_{k}[n]$ and one radial distortion $r_{k}[n]$.

In a narrowband system, where the term $I_{k}[n, \ell]$ can vary significantly, the radial distortion is prominent. In a wideband system however, the radial distortion is negligible because of Theorem 2 . When only the circularly symmetric error is present, the error due to quantization has the same distribution in the time and frequency domain and there is no difference in the performance between singlecarrier and OFDM transmission. The radial distortion, however, is easier to equalize if the symbols are in the time domain than if they are in the frequency domain. This can be seen in Figure 3. In the wideband system, we see that single-carrier and OFDM perform the same: the variance and distribution of the quantization noise is the same. In the narrowband system, the radial error due to quantization is not negligible, because the interference power $I_{k}[n, \ell]$ varies between estimates. It can be seen that the symbols of the single-carrier system is easier to distinguish than those of the OFDM system. We also see that the error variance due to quantization can be smaller in a wideband system than in a narrowband system with the same amount of antennas at the base station, at least if OFDM is used.

\section{NUMERICAL EXAMPLES}

To verify the feasibility of OFDM, we have done a numerical study of a massive MIMO system that uses linear receive combining. The

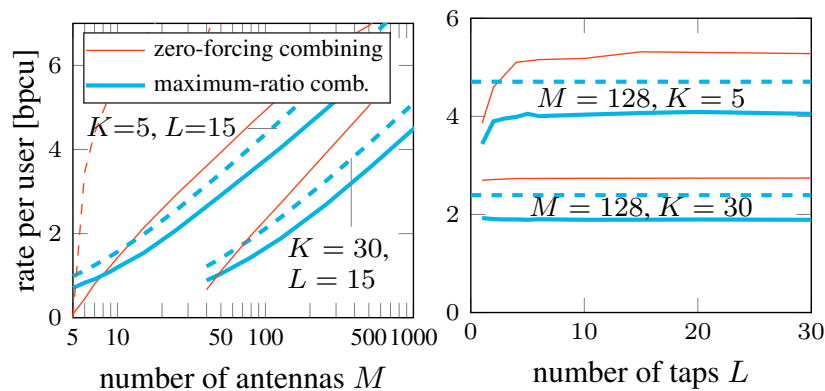

Fig. 4. The rate in a system with $M$ antennas that serves $K$ users over an $L$-tap Rayleigh fading channel. The power is $P_{k} / N_{0}=$ $10 \mathrm{~dB}, \forall k$. The curves for single-carrier and OFDM transmission coincide. The dashed curve shows the performance of the same system with perfect quantization (the rate of unquantized ZFC is mostly outside the drawn range).

channel has been modeled as IID Rayleigh with a constant power delay profile, i.e., $h_{m k}[\ell] \sim \mathcal{C N}(0,1 / L)$. The symbol estimate can be divided into

$$
\hat{s}_{k}[n]=a s_{k}[n]+z_{k}^{\prime}[n],
$$

where the choice $a=\mathrm{E}\left[s_{k}^{*}[n] \hat{s}_{k}[n]\right]$ minimizes the variance of the noise term $z_{k}^{\prime}[n]$ [17]. Then we can define a Signal-to-InterferenceQuantization-and-Noise Ratio as

$$
\mathrm{SIQNR} \triangleq \frac{|a|^{2}}{\mathrm{E}\left[\left|z_{k}^{\prime}[n]\right|^{2}\right]} .
$$

Because $\mathrm{E}\left[s_{k}^{*}[n] z_{k}^{\prime}[n]\right]=0$, we can achieve the following rate by treating all uncorrelated noise $z_{k}^{\prime}[n]$ as Gaussian [18]

$$
R \triangleq \log _{2}(1+\mathrm{SIQNR}) \quad[\mathrm{bpcu}] .
$$

This rate was computed for different numbers of antennas and different numbers of channel taps in Figure 4. It can be seen that the rate increases as the number of antennas increases and that one-bit ADCs with linear combining becomes feasible in the massive MIMO regime. Furthermore, we see that the rate also increases with the number of channel taps. The improvement saturates when $I_{k}[n, \ell]$ becomes deterministic. With a large $K$, this happens sooner, compare the improvement when $K=5$ and $K=30$ as $L$ grows in Figure 4. This suggests that one-bit ADCs perform the same or better in frequency-selective channels compared to frequency-flat channels, and that wideband systems even can improve the performance of one-bit ADCs.

\section{CONCLUSION}

In wideband massive MIMO systems, one-bit ADCs affect the performance of single-carrier and OFDM transmission in the same way, which means that many results for single-carrier systems carry over also to OFDM systems. The frequency selectivity of the wideband channel helps to spread the effect of the quantization, so that all symbols are affected in the same way. We proved that this makes the estimates of linear combiners consistent and the noise circularly symmetric Gaussian, which makes OFDM easy to implement. Further, we note that the quantization noise has two parts: one radial and one additive circularly symmetric Gaussian. Only the latter is significant in a wideband system, where we have shown that the frequency selectivity of the channel makes radial distortion negligible. 


\section{REFERENCES}

[1] R. H. Walden, "Analog-to-digital converter survey and analysis," IEEE J. Sel. Areas Commun., vol. 17, no. 4, pp. 539-550, Apr. 1999.

[2] E. Björnson, M. Matthaiou, and M. Debbah, "Massive MIMO with non-ideal arbitrary arrays: Hardware scaling laws and circuit-aware design," IEEE Trans. Wireless Commun., vol. 14, no. 8, pp. 4353-4368, Aug. 2015.

[3] J. Choi, J. Mo, and R. W. Heath Jr., "Near maximum-likelihood detector and channel estimator for uplink multiuser massive MIMO systems with one-bit ADCs," ArXiv E-Print, July 2015, arXiv:1507.04452 [cs.IT].

[4] S. Jacobsson, G. Durisi, M. Coldrey, U. Gustavsson, and C. Studer, "One-bit massive MIMO: Channel estimation and high-order modulations," ArXiv E-Print, Apr. 2015, arXiv:1504.04540v2 [cs.IT].

[5] J. Mo and R. W. Heath Jr., "Capacity analysis of one-bit quantized MIMO systems with transmitter channel state information," IEEE Trans. Signal Process., Oct. 2015.

[6] A. Mezghani and J. A. Nossek, "On ultra-wideband MIMO systems with 1-bit quantized outputs: Performance analysis and input optimization," in Proc. IEEE Int. Symp. Inform. Theory. IEEE, June 2007, pp. 1286-1289.

[7] J. Singh, O. Dabeer, and U. Madhow, "On the limits of communication with low-precision analog-to-digital conversion at the receiver," IEEE Trans. Commun., vol. 57, no. 12, pp. 3629-3639, Dec. 2009.

[8] C. Risi, D. Persson, and E. G. Larsson, "Massive MIMO with 1-bit ADC," ArXiv E-Print, Apr. 2014, arXiv:1404.7736 [cs.IT].

[9] S. Shamai, "Information rates by oversampling the sign of a bandlimited process," IEEE Trans. Inf. Theory, vol. 40, no. 4 pp. 1230-1236, July 1994.

[10] S. Wang, Y. Li, and J. Wang, "Multiuser detection in massive spatial modulation MIMO with low-resolution ADCs," IEEE Trans. Wireless Commun., vol. 14, no. 4, pp. 2156-2168, Apr. 2015.

[11] C. Studer and G. Durisi, "Quantized massive MU-MIMOOFDM uplink," ArXiv E-Print, Sept. 2015, arxiv:1509.07928 [cs.IT].

[12] 3GPP TS36.141 3rd Generation Partnership Project; Technical Specification Group Radio Access Network; Evolved Universal Terrestrial Radio Access (E-UTRA); Base Station (BS) Conformance Testing (Release 10), 3GPP Std., Rev. V10.2.0, 2011.

[13] R. Narasimha, M. Lu, N. R. Shanbhag, and A. C. Singer, "BER-optimal analog-to-digital converters for communication links," IEEE Trans. Signal Process., vol. 60, no. 7, pp. 3683-3691, July 2012.

[14] M. T. Ivrlač and J. A. Nossek, "On MIMO channel estimation with single-bit signal-quantization," in Proc. ITG Workshop Smart Antennas, 2007.

[15] O. Dabeer and U. Madhow, "Channel estimation with lowprecision analog-to-digital conversion," in Proc. IEEE Int Conf. Commun. IEEE, May 2010, pp. 1-6.
[16] H. Q. Ngo, E. G. Larsson, and T. L. Marzetta, "Energy and spectral efficiency of very large multiuser MIMO systems," IEEE Trans. Commun., vol. 61, no. 4, pp. 1436-1449, Feb. 2013.

[17] S. M. Kay, Fundamentals of Statistical Signal Processing, Volume I: Estimation Theory. Prentice Hall, 1993.

[18] M. Médard, "The effect upon channel capacity in wireless communications of perfect and imperfect knowledge of the channel," IEEE Trans. Inf. Theory, vol. 46, no. 3, pp. 933-946, May 2000 